\title{
Smarr formula for BTZ black holes in general three-dimensional gravity models
}

\author{
Chao Liang, Li Gong, and Baocheng Zhang* \\ School of Mathematics and Physics, \\ China University of Geosciences, Wuhan 430074, China
}

\begin{abstract}
Recent studies have presented the interpretation of thermodynamic enthalpy for the mass of BTZ black holes and the corresponding Smarr formula. All these are made in the background of three-dimensional (3D) general relativity. In this paper, we extend such interpretation into general 3D gravity models. It is found that the direct extension is unfeasible and some extra conditions are required to preserve both the Smarr formula and the first law of black hole thermodynamics. Thus, BTZ black hole thermodynamics enforces some constraints for general 3D gravity models, and these constraints are consistent with all previous discussions.
\end{abstract}

*Electronic address: zhangbc.zhang@yahoo.com 


\section{INTRODUCTION}

Smarr formula [1], together with the first law of black hole thermodynamics [2], plays an important role in black hole physics. For black hole solutions with a non-vanishing cosmological constant, in order to maintain the scaling relation of Smarr formula, the cosmological constant is considered as thermodynamic pressure [3 9], and the black hole mass, which is usually regarded as analogous thermal internal energy in black hole thermodynamics, is interpreted as a gravitational analog of thermodynamic enthalpy $H[10]$, i.e. $M=H=U+P V$, where $U$ is the internal energy and the pressure $P$ is derived from the cosmological constant. This has been investigated for many different situations (see Ref. [11] and references therein), and the consistent expressions for homogeneous Smarr formula and the first law have been constructed, i.e. for a $D$-dimensional singly-rotating black hole,

$$
\begin{gathered}
d M=T d S+\Omega d J+V d P, \\
(D-3) M=(D-2) T S+(D-2) \Omega J-2 V P,
\end{gathered}
$$

where $T$ is its temperature, $S$ is its entropy, $J$ is its angular momentum, $\Omega$ is its angular velocity, $V$ is its thermodynamic volume, and $P=-\frac{\Lambda}{8 \pi}=\frac{(D-2)(D-1)}{16 \pi l^{2}}$ is its pressure.

Recently, such interpretation as presented in Eqs. (1) and (2) has also been investigated for lower-dimensional black holes under the background of extended thermodynamic phase space, or "black hole chemistry" [12]. For three-dimensional (3D) Banados-TeitelboimZanelli (BTZ) black holes [13] which is an solution of 3D Einstein field equation plus a negative cosmological constant, it has been confirmed [12, 14] that Eqs. (11) and (2) can be satisfied simultaneously by assuming the negative cosmological constant as a variable thermodynamic parameter. It was found that the extension to the charged BTZ black holes is direct [12], but to ensure the Reverse Isoperimetric Inequality [8, 15] a new thermodynamic work term associated with the mass-renormalization scale has to be introduced. Moreover, the mass of BTZ black holes as the enthalpy of the spacetime was also found to be valid even under the condition of quantum correction [5].

It is realized that the preservation of Eqs. (11) and (2) was only investigated under 3D general relativity (GR), however. 3D GR propagates no physical modes (it has no local degrees of freedom) and can be formulated as a Chern-Simons (CS) gauge theory [16]. The attempt to construct generalizations [17-22] of 3D GR that propagate gravitons has 
been a revival of interest in past years. In these new 3D gravity models, some of the thermodynamic parameters for BTZ black holes which almost solves all the 3D gravity models have to be modified, and the modified parameters still satisfied the first law of black hole thermodynamics and the integral mass formula [23] but without forcing the cosmological constant into a variable. Of course, the previous Smarr-like formula, i.e. $M=\frac{1}{2} T S+\Omega J$, does not satisfy the scaling relation as presented in Eq. (2). In this paper, we expect to investigate the relations among the thermodynamic parameters under the background of extended 3D gravity models, and see if there are the same relation as that in Eqs. (11) and (2) when considering the cosmological constant as a new variable thermodynamic parameter. Moreover, a motivation to make such investigation is that there are the phenomena of continuous phase transition [24-26] for BTZ black holes in general 3D gravity models.

The organization of this paper is as follows. First, we revisit the interpretation of thermodynamic enthalpy for the mass of BTZ black holes and the homogeneous Smarr formula in the background of 3D normal Einstein gravity in the second section. In the third section, we extend these discussions to the case of 3D exotic Einstein gravity. Then, we investigate these relations systematically in general 3D gravity models and see if they are still valid, and investigate the phase transition for this case in the fourth section. Finally, we summarize our conclusion in the fifth section.

\section{NORMAL BTZ BLACK HOLES}

The two-parameter BTZ solution represents a rotating black hole in 3D spacetime with a negative cosmological constant, and its metric takes the form

$$
d s^{2}=-N^{2} d t^{2}+N^{-2} d r^{2}+r^{2}\left(N^{\phi} d t+d \phi\right)^{2}
$$

where $\phi$ is an angle with the period $2 \pi$ as the identification of the black hole spacetime. The functions $N^{2}$ and $N^{\phi}$ are

$$
N^{2}=-8 G m+\frac{r^{2}}{\ell^{2}}+\frac{16 G^{2} j^{2}}{r^{2}}, N^{\phi}=\frac{4 G j}{r^{2}}
$$

where $G$ the 3D Newton constant. Its Killing horizons are found by setting $N^{2}=0$; this gives

$$
r_{ \pm}=\sqrt{2 G \ell(\ell m+j)} \pm \sqrt{2 G \ell(\ell m-j)}
$$


We may assume without loss of generality that $j \geqslant 0$ and assume that $\ell m \geqslant j$, to ensure the existence of an event horizon at $r=r_{+}$. Thus, the parameters $m$ and $j$ can be expressed as

$$
m=\frac{r_{+}^{2}+r_{-}^{2}}{8 G \ell^{2}}, \quad j=\frac{r_{+} r_{-}}{4 G \ell} .
$$

And the temperature $T$ and the angular velocity $\Omega$ take the values

$$
T=\frac{r_{+}^{2}-r_{-}^{2}}{2 \pi r_{+} \ell^{2}}, \quad \Omega=\frac{r_{-}}{\ell r_{+}}
$$

which are independent on the concrete 3D gravity models and present the geometric properties for the 3D BTZ spacetime background.

The BTZ metric can be solved in the normal Einstein gravity with the Lagrangian

$$
L_{G}=\frac{1}{8 \pi G}\left(e_{a} R^{a}+\frac{\Lambda_{0}}{6} \epsilon^{a b c} e_{a} e_{b} e_{c}\right),
$$

where the Lagrangians are given with 3 -forms in which $e^{a}(a=0,1,2)$ is the dreibein 1forms, $\omega^{a}$ is Lorentz connection 1 -forms, $\Lambda_{0}=-\frac{1}{\ell^{2}}$ is the negative cosmological constant and $R^{a}=d \omega^{a}+\frac{1}{2} \epsilon^{a b c} \omega_{b} \omega_{c}$ is the curvature 2-form field strengths. With the BTZ metric (3) the thermodynamic parameters for normal BTZ black holes are

$$
M_{G}=m, \quad J_{G}=j, \quad S_{G}=\frac{\pi r_{+}}{2 G} .
$$

It is easy to confirm that the usual thermodynamic relations, $d M_{G}=T d S_{G}+\Omega d J_{G}$ and $M_{G}=\frac{1}{2} T S_{G}+\Omega J_{G}$, hold in this situation, but the mass formula obviously violates the scaling relation required in the Smarr formula of rotating black holes. In order to rectify this situation, it is possible to promote the cosmological constant to a variable thermodynamic parameter, so that the corresponding relations from Eqs. (1) and (2) can be reexpressed as

$$
\begin{aligned}
d M_{G} & =T d S_{G}+\Omega d J_{G}+V_{G} d P \\
0 & =T S_{G}+\Omega J_{G}-2 V_{G} P
\end{aligned}
$$

where the pressure is $P \equiv \frac{1}{8 \pi G \ell^{2}}$ and the conjugate thermodynamic volume is $V_{G}=\frac{\partial M_{G}}{\partial P}=$ $\pi r_{+}^{2}$ obtained by the mass $M\left(S_{G}, J_{G}, P\right)=\frac{4 P S_{G}^{2}}{\pi}-\frac{\pi^{2} J_{G}^{2}}{128 S_{G}^{2}}$. It is noted that the thermodynamic volume is just the $3 \mathrm{D}$ geometric volume, independent on other thermodynamic parameters except the entropy.

Like AdS black holes in higher dimensions [10], the mass of BTZ black holes should be understood as thermodynamic enthalpy when the negative cosmological constant is considered as a thermodynamic variable, but it is noted that the thermodynamic enthalpy is zero 
here. This can be understood by the fact [30] that the energy required to form the BTZ black hole is balanced by the energy required by the external forces to place the black hole into the cosmological environment.

\section{EXOTIC BTZ BLACK HOLES}

The BTZ metric can also be solved in the exotic Einstein gravity [27] with the Lagrangian

$$
L_{E}=\frac{\ell}{8 \pi G}\left[\omega_{a}\left(d \omega^{a}+\frac{2}{3} \epsilon^{a b c} \omega_{b} \omega_{c}\right)-\frac{1}{\ell^{2}} e_{a} T^{a}\right],
$$

where the torsion tensor is $T^{a}=d e^{a}+\epsilon^{a b c} \omega_{b} e_{c}$, but the corresponding thermodynamic parameters becomes

$$
M_{E}=j / \ell, \quad J_{E}=\ell m, \quad S_{E}=\frac{\pi r_{-}}{2 G}
$$

This presents some exotic properties: the metric is the same as that of 3D Einstein gravity but with reversed roles for mass and angular momentum, and the entropy is proportional to the length of the inner horizon instead of the event horizon. The corresponding understanding for these properties refer to Refs. [28, 29]. It is easy to check that the usual thermodynamic relations, $d M_{E}=T d S_{E}+\Omega d J_{E}$ and $M_{E}=\frac{1}{2} T S_{E}+\Omega J_{E}$, also hold in this situation.

As done for normal BTZ black holes, we also promote the cosmological constant into a variable in this case to see if the Smarr formula can also obtained like before. Based on this, now we express the mass of exotic BTZ black holes as

$$
M_{E}=\frac{1}{\ell}\left(\frac{2 G S_{E}^{2} J_{E}}{\pi^{2} \ell}-\frac{G^{2} S_{E}^{4}}{\pi^{4} \ell^{2}}\right)^{\frac{1}{2}}
$$

When considering the pressure $P \equiv \frac{1}{8 \pi G \ell^{2}}$, the conjugate thermodynamic volume is obtained as

$$
\begin{aligned}
V_{E} & =\frac{\partial M_{E}}{\partial P}=\frac{\partial M_{E} / \partial \ell}{\partial P / \partial \ell} \\
& =-\frac{3}{2 \ell^{2}}\left(\frac{2 G S_{E}^{2} J_{E}}{\pi^{2} \ell}-\frac{G^{2} S_{E}^{4}}{\pi^{4} \ell^{2}}\right)^{\frac{1}{2}}+\frac{1}{2 \ell^{2}}\left(\frac{2 G S_{E}^{2} J_{E}}{\pi^{2} \ell}-\frac{G^{2} S_{E}^{4}}{\pi^{4} \ell^{2}}\right)^{-\frac{1}{2}} \\
& =\frac{\pi}{2} \frac{3 r_{+}^{2} r_{-}-r_{-}^{3}}{r_{+}}
\end{aligned}
$$


where the last step uses the relation in Eqs. (6) and (12). It is noted that the exotic volume is not the geometric volume $V=\pi r_{+}^{2}$ and it also depends on the thermodynamic parameters $J_{E}$ and $P$ besides the entropy $S_{E}$.

With the expressions of thermodynamic pressure and volume, we obtain the relations again

$$
\begin{gathered}
d M_{E}=T d S_{E}+\Omega d J_{E}+V_{E} d P, \\
0=T S_{E}+\Omega J_{E}-2 V_{E} P
\end{gathered}
$$

which presents the homogenous Smarr relation.

On the other hand, one might want to make the thermodynamic volume unchanged, i.e. $V_{E}=V=\pi r_{+}^{2}$, whose thoughts stemmed from the geometric expressions for the temperature and the angular velocity. Thus the Smarr formula is written as

$$
0=T S_{E}+\Omega J_{E}-2 V P_{E}
$$

where a calculation leads to the pressure $P_{E}=\frac{3 r_{+}^{2} r_{-}-r_{-}^{3}}{16 \pi G \ell^{2} r_{+}^{2}}$. But unfortunately, this cannot preserve the first law, i.e. $d M_{E} \neq T d S_{E}+\Omega d J_{E}+V d P_{E}$, which can be obtained only through the calculation of the derivative to the AdS radius $\ell$. On the other hand, such expression for the pressure implies that $P_{E}$ is not an independent variable relative to the entropy and the angular momentum. This conflicts with the requirement of differential form of the first law of black hole thermodynamics.

\section{BTZ BLACK HOLES IN GENERAL 3D GRAVITY MODELS}

As seen above for the normal and exotic BTZ black holes that the extensive thermodynamic variables are model-dependent, so in general 3D gravity models, the mass and the angular momentum are expressed as

$$
\begin{aligned}
M & =a m+b j / \ell, \\
J & =a j+b \ell m, \\
S & =\frac{\pi}{2 G}\left(a r_{+}+b r_{-}\right),
\end{aligned}
$$

where $a$ and $b$ are the parameters dependent on the concrete models, i.e. for normal Einstein gravity, $a=1, b=0$; for exotic Einstein gravity, $a=0, b=1$. If the parameters $a$ and $b$ 
are independent on the cosmological constant or the AdS radius $\ell$, we can get the same expressions

$$
\begin{gathered}
d M=T d S+\Omega d J+V d P, \\
0=T S+\Omega J+2 V P,
\end{gathered}
$$

with the thermodynamic volume

$$
V=\frac{2 a r_{+}^{3}+3 b r_{+}^{2} r_{-}-b r_{-}^{3}}{2 r_{+}} \pi
$$

which is not geometric, but will recover geometric volume when $a=1, b=0$. It is also obvious that when $a=0, b=1, V=V_{E}$.

Then, in general 3D gravity models, are the parameters $a$ and $b$ independent on the AdS radius? As well-known, BTZ black holes are the solutions almost for all the 3D gravity models which are usually modified by topological terms, i.e. topological massive gravity (TMG) [17], minimal massive gravity (MMG) [22], which make the theory has one gravitational propagating mode, or by higher-order derivative terms, i.e. new massive gravity (NMG) [19], zwei-dreibein gravity (ZDG) [21], which make the theory have two gravitational propagating modes, or by the two terms simultaneously, i.e. general massive gravity (GMG) [20], general minimal massive gravity (GMMG) [31], or by other terms, i.e. Mielke-Baekler (MB) model [18], et al. But for almost all these modified 3D gravity models, either $a$ or $b$ or both of them are dependent on the AdS radius. Thus, we have to check if the homogenous Smarr formula is still valid when taking $a=a(\ell), b=b(\ell)$.

Assuming the Smarr formula in Eq. (18) holds, we can obtain the same expression for thermodynamic volume as in Eq. (19) but the parameters $a$ and $b$ are now the function of AdS radius $\ell$. For the first law of black hole thermodynamics, we have

$$
\frac{d M}{d \ell}=\frac{r_{+}^{2}+r_{-}^{2}}{8 G \ell^{2}} \frac{d a}{d \ell}+\frac{r_{+} r_{-}}{4 G \ell^{2}} \frac{d b}{d \ell}-\frac{r_{+}^{2}+r_{-}^{2}}{4 G \ell^{3}} a-\frac{r_{+} r_{-}}{2 G \ell^{3}} b
$$

and

$$
\begin{aligned}
T \frac{d S}{d \ell} & =\frac{r_{+}^{2}-r_{-}^{2}}{4 G \ell^{2}} \frac{d a}{d \ell}+\frac{r_{-}\left(r_{+}^{2}-r_{-}^{2}\right)}{4 G \ell^{2} r_{+}} \frac{d b}{d \ell} \\
\Omega \frac{d J}{d \ell} & =\frac{r_{-}^{2}}{4 G \ell^{2}} \frac{d a}{d \ell}+\frac{r_{-}\left(r_{+}^{2}+r_{-}^{2}\right)}{8 G \ell^{2} r_{+}} \frac{d b}{d \ell}-\frac{r_{-}^{2}}{4 G \ell^{3}} a-\frac{r_{-}\left(r_{+}^{2}+r_{-}^{2}\right)}{8 G \ell^{3} r_{+}} b \\
V \frac{d P}{d \ell} & =-\frac{r_{+}^{2}}{4 G \ell^{3}} a-\frac{r_{-}\left(3 r_{+}^{2}-r_{-}^{2}\right)}{8 G \ell^{3} r_{+}} b .
\end{aligned}
$$


Thus, it is easy to see that

$$
\frac{d M}{d \ell} \neq T \frac{d S}{d \ell}+\Omega \frac{d J}{d \ell}+V \frac{d P}{d \ell}
$$

which indicates that it is impossible to find the proper thermodynamic volume to maintain the first law and the homogenous Smarr formula simultaneously if the cosmological constant is taken as the pressure $P \equiv \frac{1}{8 \pi G \ell^{2}}$. This seems that the interpretation of enthalpy was inapplicable for the 3D AdS black hole in the general gravity models.

When we deal with the concrete gravity model, however, it is found that the simultaneous preservation of the first law and the homogeneous Smarr formula will give some further constraints when the parameters $a$ and $b$ are dependent on the AdS radius. But whether the constraints are reasonable has to be checked. Here we take the GMMG [31] for example to study this and start with its Lagrangian

$$
L_{M}=L_{G}+\frac{1}{2 \mu} L_{C S}+\frac{1}{m^{2}} L_{H}+h_{a} T^{a}+\frac{\alpha}{2} \epsilon^{a b c} e_{a} h_{b} h_{c}
$$

where $L_{C S}=\frac{1}{8 \pi G} \omega_{a}\left(d \omega^{a}+\frac{2}{3} \epsilon^{a b c} \omega_{b} \omega_{c}\right)$ is the topological modification term, $L_{H}=$ $\frac{1}{8 \pi G}\left(f^{a} R_{a}+\frac{1}{2} \epsilon^{a b c} e_{a} f_{b} f_{c}\right)$ is the higher-order derivative modification term, and $\mu, m, \alpha$ are the parameters which are introduced in TMG, NMG and MMG. For GMMG model, it was obtained [32]

$$
a=1+\frac{\gamma}{2 \mu^{2} \ell^{2}}+\frac{s}{2 m^{2} \ell^{2}}, \quad b=\frac{1}{\mu \ell},
$$

where $\gamma, s$ are the constants. Through a tedious calculation, it is found that only if

$$
\mu \ell=\text { constant and } m \ell=\text { constant }
$$

we can ensure both the first law and the homogeneous Smarr formula, which means that the interpretation of thermodynamic enthalpy for BTZ black holes in general gravity models enforces the extra constraints as in Eq. (24) when we promote the cosmological constant into a variable thermodynamic parameter.

Now we try to understand why there is such constraints as in Eq. (24). First, these constraints are consistent with the previous discussions in different 3D gravity models, in particular for some special situations, i.e. $\mu \ell=1$ in TMG model leads to 3D chiral gravity [33]; $m^{2} \ell^{2}=\frac{1}{2}$ ( or $\left.\Lambda_{0} / m^{2}=-1\right)$ in NMG leads to an extra gauge symmetry at the linearized level which allows massive modes to become partially massless [34].

On the other hand, the BTZ metric is locally isomorphic to the AdS vacuum, so any theory of 3D gravity admitting an AdS vacuum will also admit BTZ black holes. Now 
we search for the AdS vacuum for GMMG models which is also the maximally symmetric vacuum [35] of GMMG defined by

$$
G_{\mu \nu}=-\Lambda g_{\mu \nu}
$$

Thus, the GMMG field equation

$$
G_{\mu \nu}+\Lambda_{0} g_{\mu \nu}+\frac{1}{\mu} C_{\mu \nu}+\frac{\gamma}{\mu^{2}} J_{\mu \nu}+\frac{s}{2 m^{2}} K_{\mu \nu}=0
$$

where $G_{\mu \nu}$ is Einstein tensor, $C_{\mu \nu}=\frac{1}{\sqrt{-g}} \varepsilon_{\mu \alpha \beta} \nabla^{\alpha}\left(R_{\nu}^{\beta}-\frac{1}{4} \delta_{\nu}^{\beta} R\right)$ is Cotton tensor, and $J_{\mu \nu}, K_{\mu \nu}$ are the higher-order modification terms (see Ref. [33] for their expressions), will be reduced to

$$
\Lambda_{0}-\Lambda+\frac{\gamma \Lambda^{2}}{4 \mu^{2}}-\frac{s \Lambda^{2}}{4 m^{2}}=0
$$

which solves the effective cosmological constant

$$
\Lambda=\frac{2\left(1 \pm \sqrt{1-\Lambda_{0}\left(\frac{\gamma}{\mu^{2}}-\frac{s}{m^{2}}\right)}\right)}{\frac{\gamma}{\mu^{2}}-\frac{s}{m^{2}}} .
$$

It is consistent with that obtained in the linearization of GMMG field equation around the AdS metric [32]. The AdS space requires the effective cosmological constant $\Lambda=-\frac{1}{\ell^{2}}<0$. This enforces the coupling parameters to satisfy certain conditions, i.e. the minus sign for the sign " \pm " can be taken but must $\frac{\gamma}{\mu^{2}}-\frac{s}{m^{2}}>0, \Lambda_{0}<0$. Thus, we find the AdS vacuum for GMMG models with $\Lambda=-\frac{1}{\ell^{2}}$, which means that we must also find BTZ black holes because these are locally isometric to the AdS vacuum.

On the other hand, Ref. [10] has extended the Komar integral relation for the asymptotically flat black holes to that for the asymptotically AdS black holes,

$$
\frac{1}{8 \pi G} \int_{\partial \Sigma} d S_{a b}\left(\nabla^{a} \xi^{b}+2 \Lambda \omega^{a b}\right)=0
$$

where $d S_{a b}$ is the volume element normal to the co-dimension 2 surface $\partial \Sigma$ which is the boundary of the hypersurface $\Sigma$ in BTZ black hole spacetime, $\xi^{a}$ is the Killing vector on this spacetime, and $\omega^{a b}$ is the anti-symmetric Killing potential by solving $\xi^{b}=\nabla_{a} \omega^{a b}$. For the boundary $\partial \Sigma=\partial \Sigma_{\infty} \cap \partial \Sigma_{r_{+}}$, one can rearrange the Komar integral,

$$
\frac{1}{8 \pi G} \int_{\partial \Sigma_{\infty}} d S_{a b}\left(\nabla^{a} \xi^{b}+2 \Lambda \omega^{a b}\right)=\frac{1}{8 \pi G} \int_{\partial \Sigma_{r_{+}}} d S_{a b}\left(\nabla^{a} \xi^{b}+2 \Lambda \omega^{a b}\right)
$$


which leads to the homogeneous Smarr formula directly for normal BTZ black holes [12]. However, in general 3D gravity models, when we promote the cosmological constant $\Lambda$ into a variable, those coupling constants, i.e. $\mu, m$, will also become variables, as seen from Eq. (28). In order to ensure both the thermodynamic first law and the homogeneous Smarr formula to hold, one must constraint these coupling parameters to satisfy the relation in Eq. (24), which might be the justification for these constraints.

Moreover, from the left and right central charges of dual CFT [31] of GMMG model,

$$
\begin{aligned}
& C_{L}=\frac{3 \ell}{2 G}\left(1-\frac{1}{\mu \ell}+\frac{\gamma}{2 \mu^{2} \ell^{2}}+\frac{s}{2 m^{2} \ell^{2}}\right), \\
& C_{R}=\frac{3 \ell}{2 G}\left(1+\frac{1}{\mu \ell}+\frac{\gamma}{2 \mu^{2} \ell^{2}}+\frac{s}{2 m^{2} \ell^{2}}\right),
\end{aligned}
$$

the microscopic Cardy formula leads to the entropy [33, 36, 37]

$$
S=\frac{\pi^{2} \ell}{3}\left(C_{L} T_{L}+C_{R} T_{R}\right)
$$

where $T_{L}=\frac{r_{+}-r_{-}}{2 \pi \ell^{2}}, T_{R}=\frac{r_{+}+r_{-}}{2 \pi \ell^{2}}$ are the left and right temperatures of BTZ black holes respectively. Thus, we see that when the condition in Eq. (24) holds, the entropy (32) can still be regarded as independent on the AdS radius, even if the cosmological constant is promoted to a variable. This is also consistent with the requirement of differential expression for thermodynamic first law, in which the pressure $P$ and the entropy $S$ should be considered as independent variables to each other.

Finally, there exists the continuous phase transition for BTZ black holes in general 3D gravity models. This is different from that in the normal Einstein gravity, in which BTZ black holes exhibits no interesting phase behaviour [12], since its heat capacity is always positive as verified by the method of thermodynamic curvature [38]. We still take GMMG models for example. Considering the constraints (24), the heat capacity is calculated as

$$
C=\left.\frac{\partial M}{\partial T}\right|_{P, J}=\frac{4 \pi\left(a^{2}-b^{2}\right) r_{+}\left(r_{+}^{2}-r_{-}^{2}\right) \ell}{b r_{-}\left(3 r_{+}^{2}+r_{-}^{2}\right) \ell+a r_{+}\left(r_{+}^{2}+3 r_{-}^{2}\right)},
$$

where $8 G=1$ is taken for the presentation in Fig.1. It is noted that for our case, only if the parameters $a$ and $b$ have different signs, the thermodynamic process gives the interesting phase transition. 

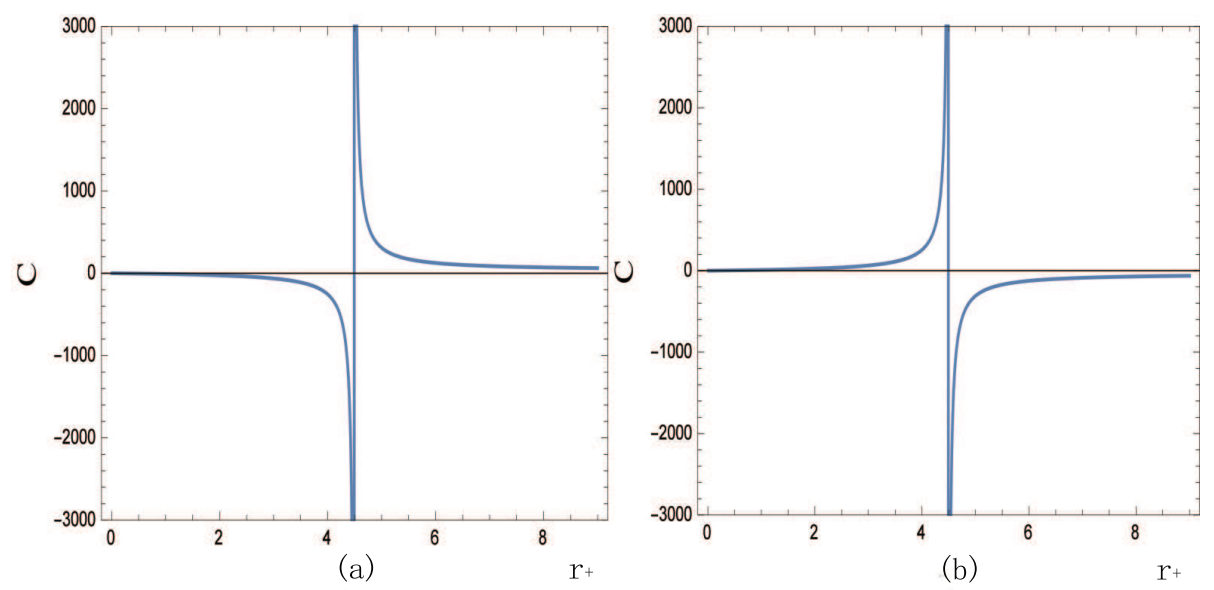

FIG. 1: The heat capacity $C$ as functions $r_{+}$for BTZ black holes in GMMG model, with $\ell=$ $1, \quad r_{-}=0.1$, while $a=-2, \quad b=3$ for (a) and $a=2, \quad b=-3$ for (b).

\section{CONCLUSION}

In this paper, we have investigated homogeneous Smarr relation and the interpretation of thermodynamic enthalpy for the mass of BTZ black holes under the background of 3D gravity theory. For normal 3D BTZ black holes, it is direct to reduce the usual relations of Eqs. (1) and (2) to 3D to obtain the corresponding Smarr formula. For exotic BTZ black holes, it is found that the thermodynamic volume is not geometric and dependent on the angular momentum and the pressure, different from the situation of normal BTZ black holes. However, when we extend these into BTZ black holes in the background of general 3D gravity models, it is impossible to find the proper conjugate thermodynamic volume when considering the variable cosmological constant as the pressure, unless some extra conditions are added. We have studied these conditions for GMMG model, and found that the conditions in Eq. (24) not only ensure both the first law and Smarr formula to hold simultaneously, but also accord with all previous discussions for GMMG model where AdS radius is not regarded as a variable. In particular, the constraint conditions are consistent with the phenomena of continuous phase transition occurred for BTZ black holes in general $3 \mathrm{D}$ gravity models. 


\section{ACKNOWLEDGE}

The authors would like to thank the support by Grant Nos. 11374330 and 91636213 of the National Natural Science Foundation of China and by the Fundamental Research Funds

for the Central Universities, China University of Geosciences (Wuhan) (No. CUG150630).

[1] Smarr L 1972 Mass Formula for Kerr Black Holes Phys. Rev. Lett. 30 71; Erratum 1973 Phys. Rev. Lett. 30521

[2] Bardeen J M, Carter B and Hawking S W 1973 The Four Laws of Black Hole Mechanics Comm. Math. Phys. 31161

[3] Creighton J D E and Mann R B 1995 Quasilocal thermodynamics of dilaton gravity coupled to gauge fields Phys. Rev. D 524569

[4] Caldarelli M M, Cognola G and Klemm D 2000 Thermodynamics of Kerr-Newman-AdS black holes and conformal field theories Class. Quant. Grav. 17399

[5] Dolan B P 2011 The cosmological constant and black-hole thermodynamic potentials Class. Quant. Grav. 28125020

[6] Dolan B P 2011 Pressure and volume in the first law of black hole thermodynamics Class. Quant. Grav. 28235017

[7] Dolan B P 2011 Compressibility of rotating black holes Phys. Rev. D 84127503

[8] Cvetic M, Gibbons G W, Kubiznak D and Pope C N 2011 Black hole enthalpy and an entropy inequality for the thermodynamic volume Phys. Rev. D 84024037

[9] Gunasekaran S, Kubiznak D and Mann R B 2012 Extended phase space thermodynamics for charged and rotating black holes and Born-Infeld vacuum polarization JHEP 1211, 110 (2012).

[10] Kastor D, Ray S and Traschen J 2009 Enthalpy and the mechanics of AdS black holes Class. Quantum Grav. 26195011

[11] Kubizňák D, Mann R B and Teo M 2016 Black hole chemistry: thermodynamics with Lambda arXiv:1608.06147.

[12] Frassino A M, Mann R B and Mureika J R 2015 Lower-dimensional black hole chemistry Phys. Rev. D 92124069 
[13] Bañados M, Teitelboim C and Zanelli J 1992 Black hole in three-dimensional spacetime Phys. Rev. Lett. 691849

[14] Wang S, Wu S, Xie F and Dan L 2006 The first law of thermodynamics of the $(2+1)$ dimensional BTZ black holes and Kerr-de Sitter spacetimes Chin. Phys. Lett. 231096

[15] Altamirano N, Kubiznak D, Mann R B and Sherkatghanad Z 2014 Thermodynamics of rotating black holes and black rings: phase transitions and thermodynamic volume Galaxies $\mathbf{2}$ 89

[16] Horne J H and Witten E 1989 Conformal Gravity in Three Dimensions as a Gauge Theory Phys. Rev. Lett. 62501

[17] Deser S, Jackiw R and Templeton S 1982 Topologically massive gauge theories Ann. Phys. (N.Y.) 140372

[18] Mielke E and Baekler P 1991 Topological gauge model of gravity with torsion Phys. Lett. A 156399

[19] Bergshoeff E A, Hohm O and Townsend P K 2009 Massive Gravity in Three Dimensions Phys. Rev. Lett. 102201301

[20] Hohm O, Routh A, Townsend P K and Zhang B 2012 On the Hamiltonian form of 3D massive gravity Phys. Rev. D 86084035

[21] Bergshoeff E A, de Haan S, Hohm O, Merbis W and Townsend P K 2013 Zwei-Dreibein Gravity: A Two-Frame-Field Model of 3D Massive Gravity Phys. Rev. Lett. 111111102

[22] Bergshoeff E, Hohm O, Merbis W, Routh A J and Townsend P K 2014 Minimal massive 3D gravity Class. Quantum Grav. 31145008

[23] Zhang B 2016 The mass formula for an exotic BTZ black hole Ann. Phys. (N.Y.) 367280

[24] Lousto C O 1995 Emergence of an effective two-dimensional quantum description from the study of critical phenomena in black holes Phys. Rev. D 511733

[25] Peca C S and Lemos J P S 1999 Thermodynamics of Reissner-Nordström-anti-de Sitter black holes in the grand canonical ensemble Phys. Rev. D 59124007

[26] Ma M, Liu F and Zhao R 2014 Continuous phase transition and critical behaviors of 3D black hole with torsion Class. Quantum Grav. 31095001

[27] Witten E $19882+1$ dimensional gravity as an exactly soluble system Nuclear Phys. B 311 46

[28] Townsend P K and Zhang B 2013 Thermodynamics of "Exotic" Bañados-Teitelboim-Zanelli 
Black Holes Phys. Rev. Lett. 110241302

[29] Zhang B 2013 Statistical entropy of a BTZ black hole in topologically massive gravity Phys. Rev. D 88124017

[30] Kubiznak D and Mann R B 2014 Black Hole Chemistry arXiv:1404.2126.

[31] Setare M R 2015 On the generalized minimal massive gravity Nucl. Phys. B 898259

[32] Setare M R and Adami H 2015 Black hole conserved charges in Generalized Minimal Massive Gravity Phys. Lett. B $\mathbf{7 4 4} 280$

[33] Li W, Song W and Strominger A 2008 Chiral gravity in three dimensions JHEP 04082

[34] Deser S, Ertl S and Grumiller D J. 2013 Canonical bifurcation in higher derivative, higher spin, theories J Phys. A: Math. Theor. 46214018

[35] Bergshoeff E A, Hohm O and Townsend P K 2009 More on massive 3D gravity Phys. Rev. D 79124042

[36] Strominger A 1998 Black hole entropy from near-horizon microstates JHEP 02009

[37] Saida H and Soda J 2000 Statistical entropy of BTZ black hole in higher curvature gravity Phys. Lett. B 471358

[38] Cai R G and Cho J H 1999 Thermodynamic curvature of the BTZ black hole Phys. Rev. D 60067502 